\title{
Large Data Management in loT Application for Supply Chain Environment
}

\author{
Bhavana M. Bachhav \\ Department of Computer Engineering \\ SKNCOE, Vadgaon (BK), Pune-411041
}

\author{
Parikshit N. Mahalle, PhD \\ Department of Computer Engineering \\ SKNCOE, Vadgaon (BK), Pune-411041
}

\begin{abstract}
In numerous IoT applications, large number of sensors and data receivers sends information to server. The servers gather information that reaches huge amount in short time. In such cases, IoT application can face the challenge of real time managing and extracting client useful information from the whole data stored on server. The main challenge faced in global supply chain management (SCM) is the development of decision making models as the huge amount of data stored on server. Here the approach is designed considering the sensor data set for analyzing all factors involved, and depending upon the analyzed data prediction of the future trade scenario is done with the help of linear regression. This helps in any manufacturing company for decisions making, systematizing their products/services, managing inventories, Prices of the product and warehousing arrangement.
\end{abstract}

\section{Keywords}

Internet of Things (IoT), Data management, Supply chain management, value Prediction

\section{INTRODUCTION}

The internet of things (IoT) is a network that connects various types of objects to the internet through different kinds of information perception devices so that all the physical objects are able to exchange information with each other. Data is one of the most valuable aspects of the IoT. The term IoT has different meaning for different people - IoT includes sensors, objects, smart devices, services etc. that can interact with user and among themselves [4]. One of the objectives of the Internet of Things (IoT) research and development is to enable real world objects to be connected to the Web, so data generated by those objects can be discovered, collected, processed, shared and utilized to create intelligent and useful applications and services in many domains such as smart cities, environment monitoring, health and energy.

From the data processing point of view, one of the challenges in managing the IoT data is how to deal with the large number of heterogeneous sensing sources in a particular application domain. If we take the smart city applications, the real world data made available to the city applications is not only from sensor networks installed by city authorities at fixed locations, but also from mobile sources such as buses and taxis equipped with environment monitoring sensors and participatory sensing from citizens smart phones.

One of the difficulties when managing the IoT information is the means by which to deal with the vast number of heterogeneous detecting objects in a specific application area , From the information handling perspective. In the event that we take the keen /smart city applications, the real time data made accessible to the city applications is not only just from sensor systems introduced by city experts at settled areas, but also from mobile objects such as buses and taxis routed with condition checking sensors and participatory detecting from nationals advanced mobile phones. The lifecycle of data within an IoT system is illustrated in Fig 1. Querying and analysis are the end points that initiate and consume data production [3]. We divide an IoT data management system based on the data lifecycle into an online frontend that interacts directly with the interconnected IoT objects and sensors, and an offline backend that handles the mass storage and in-depth analysis of IoT data.

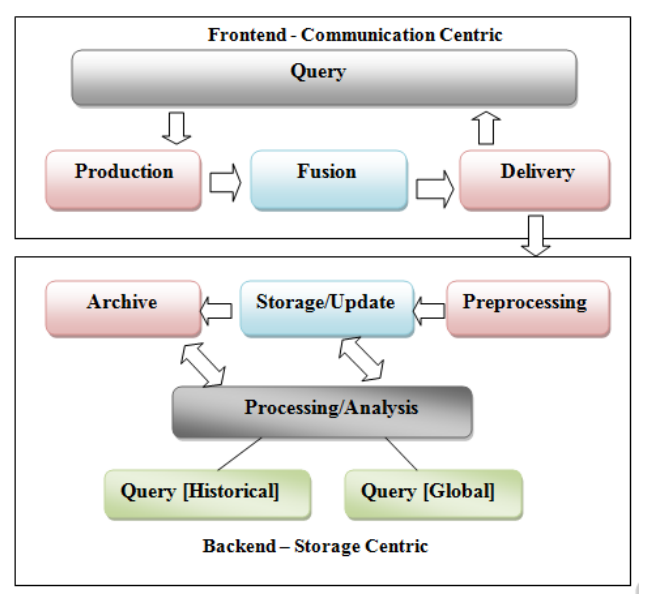

Fig. 1. Data lifecycle and management in IoT

Supply Chain Management (SCM) isthe administration and control of all materials and data in the coordinations procedure from obtaining of crude materials to conveyance to the end client. The vast majority of organizations rate SCM and stock control as the way 
to their company's future achievement, even survival. The supply chain envelopes all activities, envelopes all exercises related with the trnasformation and change of goods from the crude/raw materials organize, all the way to the nish client, and also the related data streams. The objective of supply chain network are multidimensional and include cost minimization, improved communication among supply chain companies,increased levels of service, and increased exibility in terms of response time and delivery.

\section{MOTIVATION}

In most of the IoT application, large number of sensors and data receivers sends information to server. The server gathers the information that later on becomes the huge amount in short time. IoT application faces the challenge of real time managing/extracting client useful information from whole data stored on server. Considering SCM scenario, the manufacturing industries have huge amount of data to be stored on the server. In such cases, manufacturer can face the challenge in monitoring the real time data based on which manufacturer companies can predict the future values and take decision about quantity, selling performance, production planning and cost. The proposed system helps in take manufacturing decisions in SCM that helps in improving growth of manufacturing industries.

\section{LITERATURE SURVEY}

[1] Catalin Constantin CERBULESCU, Claudia Monica ,Large data management in IoT application, ,2016 IEEE,978-1-46738606-7., focuses that IOT applications can face the challenge of real time managing or displaying or extracting client useful information from the whole data stored on servers.

[2] Abu-Elkheir, M., Data management for the Internet of Things: Green directions, Globecom Workshops (GC Wkshps), 2012 IEEE, Anaheim,CA, pp 386 390, 3-7 Dec. 2012, focuses on the life cycle of data within the Internet of Things and survey the current research in the data management field for the Internet of Things has discussed.

[3] Mervat Abu-Elkheir, Mohammad Hayajneh and Najah Abu Ali ,'Data Management for the Internet of Things: Design Primitives and Solution",Sensors 2013, 13, 15582-15612; doi:10.3390/s131115582,14 November 2013 ,ISSN 1424-8220, focuses on the survey of the data management solutions that are proposed for IoT or subsystems of the IoT has been done. The distinctive design primitives are highlighted.

[4] Yuchao Zhou, Suparna De ; Wei Wang ; Klaus Moessner, Enabling Query of Frequently Updated Data from Mobile Sensing Sources, Inst. for Commun. Syst., Univ. of Surrey, Guildford, UK, Computational Science and Engineering (CSE), 2014 IEEE 17th International Conference on, 19-21 Dec. 2014, Page(s): 946 952, ISBN: 978-1-4799-7980-6, focuses on two problems: (i) how to design a common, structured sensing layer for the heterogeneous, mobile data sources and, (ii)how to query FUTS (Frequently Updated, Time stamped and Structured) data from these sources.

[5] Xingjun Hao, X. Hao, Peiquan Jin ; Lihua Yue, Efficient Storage of Multi-Sensor Object-Tracking Data, School of Computer
Science and Technology, University of Science and Technology of China, Hefei, IEEE Transactions on Parallel and Distributed Systems (Volume:PP ,Issue: 99 ), Page(s): 1-5 ISSN : 1045-9219 , proposed the first read/write-optimized solution for storing multi-sensor object-tracking data on HDFS.

[6] Ting Lu, Jun Fang, Cong Liu, "A Unified Storage and Query Optimization Framework for Sensor Data", 2015 12th Web Information System and Application Conference (WISA), 11-13 Sept. 2015, 229 -234, ISBN: 978-1-4673-9371-3, proposed that Traditional data storage and query approaches cannot handle large amount of sensor data properly. To deal with such limitations, a unified storage and query optimization framework, named DeCloud-RealBase, is proposed towards the management of large volumes of sensor data.

[7]Tingli LI, Yang LIU, Ye TIAN, Shuo SHEN, Wei MAO, A storage solution for massive IoT data based on NoSQL , 978-07695 -4865-4/12 , 2012 IEEE DOI 10.1109/GreenCom.2012.18, proposed a storage management solution called IOTMDB based on NoSQL as current storage solutions are not performing well support storing massive and heterogeneous data collected by IoT devices. Some evaluations are also carried out instead of considering only about expressing and organizing of IoT data.

[9]Susanna Spinsante, Ennio Gambi, Laura Montanini, Laura Raffaeli, Data management in ambient assisted living platforms approaching IoT : a case study,978-1-4673-9526-7/15 2015 IEEE, analyzes the issues related to data management starting from a review of state of art for drawing a general approaches. In this paper, investigation has been done on data handling and management issues from the adoption of IoT paradigm in ambient assisted living platform.

[10]Y. Qin, Q. Z. Sheng, N. J. G. Falkner, S. Dustdar, H. Wang, and A.V. Vasilakos, When things matter: A data centric view of the Internet of Things, main techniques in IoT from data centric point of view, which includes data stream processing, data storage models , complex event processing and searching has been discussed.

\section{GAP ANALYSIS}

The classification of data within IoT is a useful task. A few information is discrete and some continuous, some naturally produced and somewhere in the range of a contribution by people. Various data format, type of storage for generated data, architecture used, different processing speed and server response are the points which are taken into consideration for gap analysis .Here in fig. 2 , we have classified the information into the accompanying areas: RFID, address/special identifiers, clear information, positional and ecological information, sensor information, veriable/historical information. Radio Frequency Identification refers to distinguishing proof and following utilizing radio waves and is turning into a typical place innovation. In paper [1],[3],[5],[7],[8],[10], sensor data is used where in [2] and [6] time series data and in[4],[9]historical data used. Processing speed is high in [1][2][6][7][9]where medium and low in [4],[8] and [3],[5],[10] respectively. 


\begin{tabular}{|l|l|l|l|l|l|}
\hline $\begin{array}{l}\text { Paper } \\
\text { no }\end{array}$ & Data format & Storage & architecture & $\begin{array}{l}\text { Processing } \\
\text { speed }\end{array}$ & $\begin{array}{l}\text { Server } \\
\text { response }\end{array}$ \\
\hline 1 & $\begin{array}{l}\text { Sensor data } \\
\text { RFID }\end{array}$ & $\begin{array}{l}\text { Cloud } \\
\text { based }\end{array}$ & Centralized & High & Good \\
\hline 2 & $\begin{array}{l}\text { Time series } \\
\text { data }\end{array}$ & Local & Decentralized & High & Good \\
\hline 3 & Sensor data & Local & centralized & Low & Good \\
\hline 4 & Historical data & $\begin{array}{l}\text { Cloud } \\
\text { based }\end{array}$ & Centralized & Medium & moderate \\
\hline 5 & Sensor data & $\begin{array}{l}\text { Cloud } \\
\text { based }\end{array}$ & Centralized & Low & Moderate \\
\hline 6 & $\begin{array}{l}\text { Time series } \\
\text { data }\end{array}$ & Local & Decentralized & High & Good \\
\hline 7 & Sensor data & Local & Centralized & High & Moderate \\
\hline 8 & Sensor data & Local & Decentralized & Medium & Good \\
\hline 9 & Historical data & $\begin{array}{l}\text { Cloud } \\
\text { based }\end{array}$ & Centralized & High & Good \\
\hline 10 & Sensor data & $\begin{array}{l}\text { Cloud } \\
\text { based }\end{array}$ & Centralized & Low & Good \\
\hline
\end{tabular}

Fig. 2. Gap Analysis

\section{MATHEMATICAL MODEL}

Let $\mathrm{S}$ be the system,

$\mathrm{S}=\{\mathrm{I}, \mathrm{O}, \mathrm{F}\}$

Let I be the input to the system,

$$
\mathrm{I}=\{\text { Sensor Data Set }\}
$$

$\mathrm{O}$ is the output of functions prediction values.

$$
\mathrm{O}=\{\mathrm{O} 1, \mathrm{O} 2, \mathrm{O} 3, \mathrm{O} 4\}
$$

O1 - Product Quantity

$\mathrm{O} 2$ - Product selling performance

O3 - Production Planning

O4 - Product Price

Let $\mathrm{F}$ be the functionality

$$
\mathrm{F}=\{\mathrm{F} 1, \mathrm{~F} 2\}
$$

F1- Data Analysis on sensor data F2 - Take Decision

$O$ be the output of linear regression for prediction of next day, month or year product value.

F2 is prediction function after data analysis

$$
\mathrm{F} 1 \longleftarrow \mathrm{F} 2
$$

$\mathrm{F} 2$ is dependent on $\mathrm{F} 1$ output value $\mathrm{O} 2, \mathrm{O} 3, \mathrm{O} 4, \mathrm{O} 5$ output depends on $\mathrm{F} 2$.

$\mathrm{O}=\{$ Product Quantity, Product selling performance, Production Planning, Product Price $\}$

\section{SYSTEM ARCHITECTURE}

Here, Fig 4. Shows the architecture of the proposed system. The working of the system is as follows:

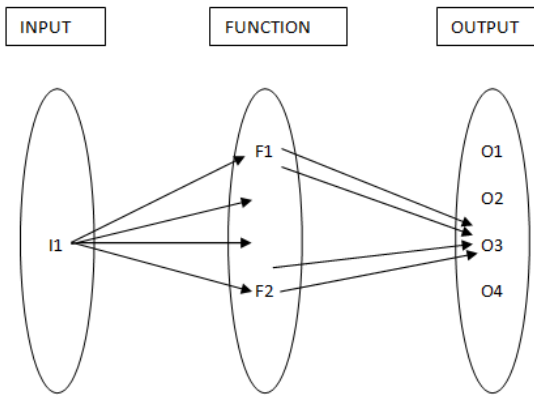

Fig. 3. Venn Diagram

Sensor data set : In wireless sensor networks (WSNs), all the data collected by the sensor nodes are forwarded to a sink node.

Data analysis : We can analyze the particular product data as well as the overall data. Linear regression algorithm on analyzed data can be done to predict the next value.River waves also predict the next value but it always changeable. So we do comparison in both algorithm and find out the algorithm that gives more accurate predicted values.

Linear regression algorithm : In this algorithm we gives the sequence wise data and predict the upcoming next value.

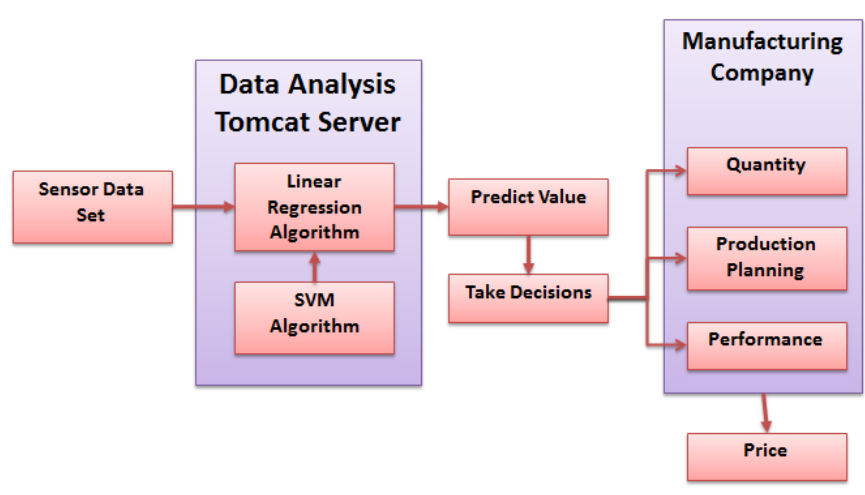

Fig. 4. System architecture of proposed work

River wave algorithm : The River Wave Algorithm have same functionality as the linear regression but it gives the changeable result.

Manufacturing company : We apply the algorithm on sensor data set for data analysis and predict the result. On the basis of this result the manufacturing company take decision to increase the company growth, and make profit. On the company history we predict the product quantity and product selling price. On the basis of this result the manufacturer has taken decision how to improve the production planning and performance. And then finally they decided the product price. 


\section{RESULTS}

1] Here, in screenshot 1, the product name is searched which we need to predict. According to that, next day, month and year as well as previous month, day and year prediction is calculated. Current sold quantity of product is also obtained. Graphs are generated for day a wise and month wise prediction.

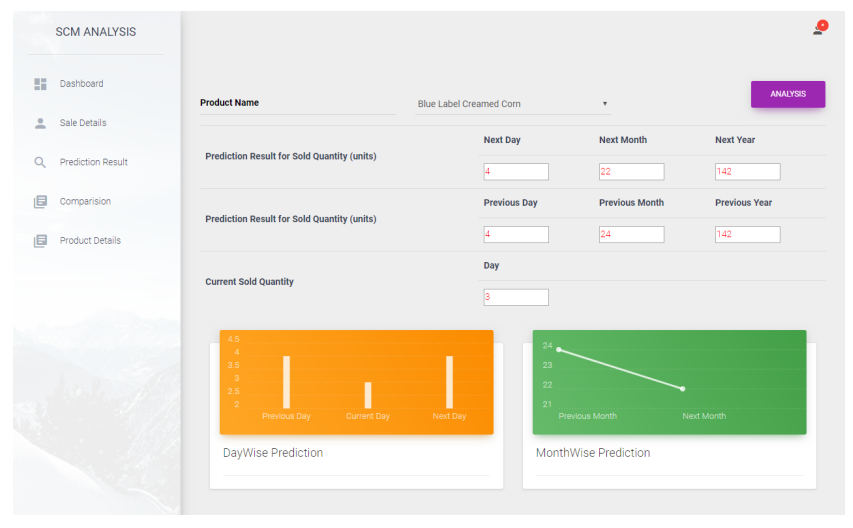

Fig. 5. Prediction on basis of sold products

2] In screenshot 2, Prediction is implemented successfully for reducing excess production of various products. This helps in reducing the total cost of products which are nally supplied to the customers. Two algorithmic comparisons is implemented here in the prediction module. Linear wave algorithm and river wave algorithm are considered.

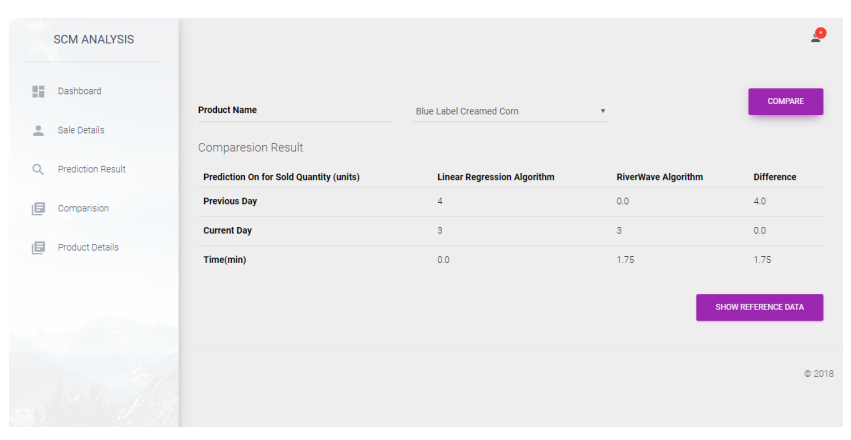

Fig. 6. Comparision

\section{CONCLUSION}

In this paper, discussion is done on the IoT data management lifecycle, in which data goes through various stages of processing. A detailed discussion is done on a analytics for Manufacturing Decisions in Supply Chain Management. It helps in taking the manufacturing decisions in SCM. The linear regression algorithm is applied on sensor data set for data analysis and depending upon on that prediction the manufacturer take the decision about how to improve the production planning and performance And then finally they decided the product price. This system can help to increase the company growth, and make profit of manufacturing industries. It predicts the data and on basis of that data manufacturer takes decisions. It helps in reducing total cost of products for the end user.

\section{REFERENCES}

[1] Catalin Constantin CERBULESCU, Claudia Monica," Large data management in IoT application ”,2016 IEEE,978-1-46738606-7.

[2] Abu-Elkheir, " Data management for the Internet of Things: Green directions ", Globecom Workshops (GC Wkshps), 2012 IEEE, Anaheim,CA, pp 386 390, 3-7 Dec. 2012

[3] "Data Management for the Internet of Things: Design Primitives and Solution", Mervat Abu-Elkheir, Mohammad Hayajneh and Najah Abu Ali,Sensors 2013, 13, 15582-15612; doi:10.3390/s131115582, 14 November 2013 ,ISSN 1424-8220

[4]Yuchao Zhou, Suparna De ; Wei Wang ; Klaus Moessner, "Enabling Query of Frequently Updated Data from Mobile Sensing Sources”, Inst. for Commun. Syst., Univ. of Surrey, Guildford, UK, Computational Science and Engineering (CSE), 2014 IEEE 17th International Conference on, 19-21 Dec. 2014, Page(s): 946 952, ISBN: 978-1-4799-7980-6

[5] Xingjun Hao, X. Hao, Peiquan Jin ; Lihua Yue, " Efficient Storage of Multi-Sensor Object-Tracking Data ", School of Computer Science and Technology, University of Science and Technology of China, Hefei, IEEE Transactions on Parallel and Distributed Systems (Volume:PP ,Issue: 99 ), Page(s): 1-5 ISSN : 1045-9219

[6] Ting Lu, Jun Fang, Cong Liu, "A Unified Storage and Query Optimization Framework for Sensor Data", 2015 12th Web Information System and Application Conference (WISA), 11-13 Sept. 2015, 229 -234, ISBN: 978-1-4673-9371-3

[7] Cooper, J.; James, " Challenges for database management in the internet of things", IETE Tech.Rev. 2009, 26, 320329.

[8] Y. Qin, Q. Z. Sheng, N. J. G. Falkner, S. Dustdar, H. Wang, and A.V. Vasilakos, "When Things Matter: A Data-Centric View of the Internet of Things,in CoRR, 2014.

[9] L. Chen, M. Tseng, and X. Lian, "Development of foundation models for Internet of Things," Frontiers of Computer Science in China, vol. 4, no.3, pp. 376-385, September 2015.

[10] K. Aberer, M. Hauswirth, and A. Salehi, "A middleware for fast and flexible sensor network deployment," in Proc. 32nd International Conference on Very Large Databases, 2006 